\section{Acute heart failure due to the left atrial myxoma causing mitral valve obstruction}

\author{
DAMIR FABIJANIĆ • CRISTIJAN BULAT • VEDRAN CAREVIĆ • \\ MATIJA MILIĆ • NINA BEROVIĆ • TONĆI BATINIĆ
}

DAMIR FABIJANIĆ $(\bowtie)$ VEDRAN CAREVIĆ • NINA BEROVIĆ DAMIR BONACIN University Hospital Split Spinčićeva 1, 21000 Split, Croatia

Phone: +38521556766

Fax: +38521556031

E-mail: damir.fabijanic@st.t-com.hr

TONĆI BATINIĆ

Department of Radiology, Split University Hospital, Split, Croatia

\begin{abstract}
A 64 year-old woman without history of cardiovascular diseases and without cardiovascular risk factors was referred to our hospital because of acute heart failure. A real-time three-dimensional echocardiography (3D) revealed left atrium tumor causing mitral valve obstruction. $3 D$ characteristics of the tumor were highly suggestive of cardiac myxoma. Surgical excision was successfully performed and myxoma was confirmed histologically. Although cardiac myxoma typically presents a silent heart failure, our case suggests that it should be even considered a cause of acute heart failure, especially in patients with no previous history or signs of cardiovascular disease.
\end{abstract}

Key words: acute heart failure, echocardiography, myxoma.

\section{Introduction}

Myxoma is the most common primary cardiac tumor. It accounts for $50 \%$ of the benign tumors of the heart. $(1,2)$ Despite its benign pathologic nature, catastrophic results can occur because of systemic or pulmonary embolism and intracardiac obstruction. $(1,2)$ Left atrial myxomas usually cause silent heart failure, and rarely acute heart failure can be the first clinical manifestation. $(1,2)$ We present a patient with acute heart failure due to the huge left atrial myxoma causing mitral valve obstruction.

\section{Case presentation}

A 64 year-old woman without history of cardiovascular diseases and without cardiovascular risk factors was referred to our hospital because of moderate chest pain, dyspnea, orthopnea and fatigability occurred one day before admission. However, the first symptoms appeared 2 months before hospitalization, in form of exertional dyspnea.
On physical examination the patient was afebrile, dyspneic and orthopneic. Cardiac auscultation revealed a regular rhythm of approximately 110 beats per minute; quiet heart sounds and $3 / 6$ diastolic murmur were audible at the apex. Blood pressure was 120/80 mm $\mathrm{Hg}$. Respiratory rate was 28 breaths per minute. Pulmonary auscultation revealed bilateral crackles in lower lung fields.

Electrocardiogram documented sinus tachycardia (100/min). Chest radiography showed normal cardiac silhouette with features of pulmonary edema: a pulmonary vascular congestion and Kerley $\mathrm{B}$ lines at the lung bases. Laboratory data revealed high erythrocyte sedimentation rate $(50 \mathrm{~mm} / \mathrm{hr})$ and C-reactive protein value $(44.8 \mathrm{mg} / \mathrm{L})$. Other hematological and biochemical parameters were within normal limits. Patient was hemodynamically stabilized. Transthoracic echocardiography showed a huge, mobile, hyperechogenic, heterogeneous, and lobulated left-atrial mass measuring approximately $60 \mathrm{~mm}$ by $40 \mathrm{~mm}$. The mass almost completely filled left atrium and was attached to the anterior leaflet of the mitral valve with wide short pedicle. In diastole, the tumor was obstructing mitral valve orifice causing a functional stenosis. Other echocardiographic parameters were unremarkable. The systolic function was preserved with an estimated left ventricular ejection fraction of $68 \%$. Real-time threedimensional (3D) transthoracic (TTE) and transesophageal (TEE) echocardiography allowed to accurately image the entire volume of the myxoma and its relationship to other intracardiac structures as well as to analyse the dynamic mitral valve obstruction (figure $1 \mathrm{~A})$. The size of the lobulated mass as assessed by $3 \mathrm{D}$ TTE was $65 \times 45 \times 22$ $\mathrm{mm}$. The mass stalk was seen attached to the basal segment of the posterior mitral valve leaflet (figure 1B). Also, 3D TEE enabled a better analysis of the tumor composition. Namely, 3D TEE reconstruction revealed heterogeneous mass with multiple necrotic and haemorrhagic zones which supported cardiac myxoma diagnosis. 


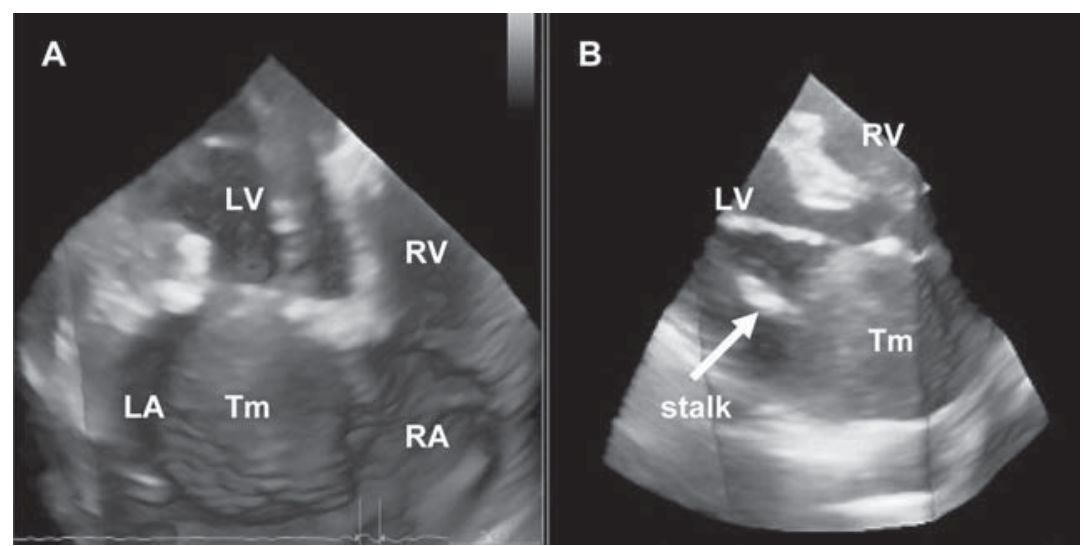

Figure 1. Three-dimensional real time transthoracic echocardiography showed tumor which almost fills left atrium (A) attached to the basal segment of the posterior mitral valve leaflet with wide short stalk (B).

LA, left atrium; LV, left ventricle; RA, right atrim; RV, right ventricle; Tm, tumor.

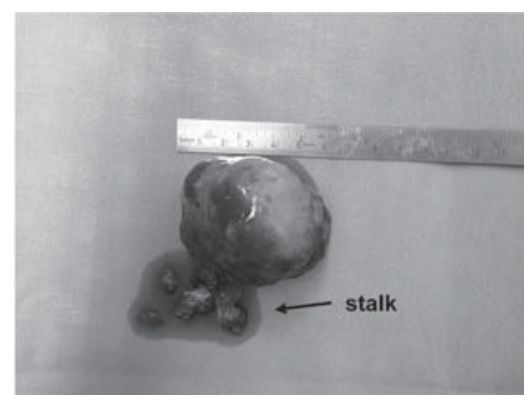

Figure 2. Completely removed tumor.

The tumor was completely surgically removed (figure 2). The histology of the specimen revealed a cardiac myxoma. Patient was discharged uneventfully on the $8^{\text {th }}$ postoperative day. Five months later, the patient remains well, with normal echocardiographic findings.

\section{Discussion}

Primary cardiac tumors are uncommon with the incidence between $0.0017 \%$ and $0.33 \%$. $(1,2)$ About two-thirds of these tumors are myxoma that typically arises in the left atrium (80\%) along the interatrial septum near the fossa ovalis. $(1,2)$ Occasionally, myxomas arise in the right atrium (15\%), the ventricles (3-4\%), or valves. Cardiac myxomas arise more detecting and distinguishing intracardiac masses. (4-7) However, TEE has advantage in detection of small masses (1 to $3 \mathrm{~mm}$ in diameter), especially in patients with suboptimal TTE images. (7) The TEE also permits a sharper picture of the attachment or stalk of the mass, and more precise characterization of its size, shape, and location. (4) Two-dimensional echocardiography measurements may underestimate the true maximum size of irregularly shaped structures. Therefore, 3D offers incremental value for the evaluation of cardiac masses by providing more accurate assessment of the size and shape of the mass as well as in some cases, composition of the mass. (8) $3 \mathrm{D}$ aids with the relationship between the mass and adjacent structures. (8) In that context, better characterization of intracardiac mass with 3D can completely eliminate the need of additional imaging techniques, especially cardiac magnetic resonance imaging and multislice computed tomography.

The accepted treatment of cardiac myxoma is operative excision. $(1,2)$ Surgical removal of a tumor is important for preventing systemic or pulmonary emboli, eliminating valvular obstruction, maintaining systolic function, and restoring biventricular diastolic function. $(1,2)$ It should not be delayed since death from obstruction to flow or embolization may occur in $8 \%$ of patients awaiting operation. (2) Surgical treatment leads to complete resolution with low rates of recurrence and good long-term survival. $(1,2)$

In conclusion, the left atrial myxomas should be considered in the differential diagnosis of acute heart failure, particularly in patients with no previous history or symptoms of cardiovascular diseases. Echocardiography provides substantial advantages in detecting cardiac masses. Therefore, echocardiography is recommended early in the work-up of acute heart failure of unknown etiology after the common causes have been ruled out. 


\section{REFERENCES}

1. Percell RL Jr, Henning RJ, Siddique Patel M. Atrial myxoma: Case report and a review of the literature. Heart Dis 2003;5:224-30.

2. Reynen K. Cardiac myxomas. N Engl J Med 1995;333:1610-7.

3. Fabijanić D, Rudež I, Kardum D, Radić M, Glavaš D, Lozo P. Pulmonary embolism due to the right atrial myxoma. Coll Antropol 2006;30:933-6.

4. Engberding R, Daniel WG, Erbel R, Kasper W, Lestuzzi C, Curtius JM, et al. Diagnosis of heart tumors by transoesophageal echocardiography: a multicentre study in 154 patients. European Cooperative Study Group. Eur Heart J 1993;14:1223-8.

5. Zekanović D, Carević V, Šušak Z. Atypical presentation of thrombosis of a permanent pacemaker lead. SIGNA VITAE 2012;7:49-51.

6. Fabijanić D, Bulat C, Letica D. Echocardiographic appearance of a hydatid cyst of the papillary muscle and chordate tendineae. J Clin Ultrasound 2011;39:431-3.

7. Fabijanić $\mathrm{D}$, Rudež I, Radić M, Unić D, Barić D, Kardum D. Pulmonary embolism due to the right atrial thrombus mimicking atrial myxoma. Chin Med J 2010;123:2483-5.

8. Tolstrup K, Shiota T, Gurudevan S, Luthringer D, Luo H, Siegel RJ. Left atrial myxomas: correlation of two-dimensional and live threedimensional transesophageal echocardiography with the clinical and pathologic findings. J Am Soc Echocardiogr 2011;24:618-24. 